\title{
Pulmonary valve sarcoma in patient with coronavirus disease of 2019 (COVID-19), mimicking pulmonary thromboembolism, a very
}

\section{rare case}

\author{
Rozita Jalalian ${ }^{1}$, Javad Sadraei ${ }^{1}$, soheil azizi ${ }^{1}$, Babak Bagheri ${ }^{1}$, and Mohammadreza \\ Iranian $^{2}$ \\ ${ }^{1}$ Mazandaran University of Medical Sciences \\ ${ }^{2}$ Iran University of Medical Sciences
}

June 14, 2021

\begin{abstract}
Pulmonary artery and pulmonary valve sarcoma are malignant and very rare vascular tumors with aggressive clinical course and very poor outcomes. Patients affected by coronavirus disease of 2019 (COVID-19) are at higher risk for thromboembolism complication. we describe young woman with history of corona virus pneumonia and progressive dyspnea, hemodynamic disturbance, edema with initial evaluation and clinical diagnosis of pulmonary thromboembolism. But further imaging study and pathology demonstrated, Giant sarcoma of pulmonary valve, obstructing pulmonary valve and extending to right ventricular outflow tract and main of pulmonary artery.
\end{abstract}

\section{Introduction:}

Pulmonary artery sarcomas (PASs) are the most common primary tumors of the pulmonary artery (PA), but the incidence of this diagnosis is extremely rare. PASs is predominantly presented among patients from their third to seventh decade, with an average age of 49 years. PASs are always highly malignant, women are involved twice as often as men. The most common clinical manifestation of PASs is dyspnea (72\%) followed by chest pain (45\%), cough (42\%), and hemoptysis (24\%). Systematic symptoms of PASs are less rare and they include weight loss $(21 \%)$, syncope $(9 \%)$, and fever $(8 \%)$. The presence of these symptoms often causes misdiagnosis of pulmonary thromboembolism (PTE) (1-3).

\section{Case report:}

A 40 years old woman presented at the emergency department because of progressive dyspnea, orthopnea, edema, weakness. She has involved with COVID-19 about 2 months ago according to polymerase chain reaction $(\mathrm{PCR})$ and chest computed tomography $(\mathrm{CT})$, But she didn't recover from respiratory symptoms in spite of adequate treatment. In admission time, she has respiratory distress, tachycardia, tachypnea, edema, cyanosis and coldness of extremely and systemic hypotension (Blood Pressure: 90/65 mmHg). O2 saturation in air room was $86 \%$ and with noninvasive $\mathrm{O} 2$ therapy increased to $89 \%$.

Electrocardiogram (ECG) showed sinus tachycardia, right axes deviation and right ventricle (RV) strain pattern in precordial leads (Figure 1). Chest $\mathrm{X}$ ray showed significant cardiomegaly, RV enlargement and prominent main of PA and left PA without evidence of pulmonary venous congestion (Figure 2). Spiral chest CT demonstrated evidence of previous COVID-19 involvement with ground glass appearance and air trapping in both lungs and also cardiomegaly and large size pericardial effusion (Figure 3). 
Transthoracic echocardiography (TTE) showed severe RV enlargement with severe RV systolic dysfunction, right atrial (RA) enlargement and abnormal interventricular septal motion. D shaped appearance of Interventricular septum in parasternal short axis (PSAX) view with mid systolic flattening, representing severe RV pressure overload and signs of severely increased RV afterload pattern. In PSAX view, very large size nonhomogeneous solid mobile mass is seen in distal right ventricular outflow tract (RVOT) extended to main $\mathrm{PA}$ and has attachment to pulmonary valve (PV). The PV obstruction was severe and blood flow to main PA was reduced. Left and right PA and pulmonary bifurcation were evaluated, that were spared without any mass. Color doppler study of tricuspid valve (TV) inflow showed severe tricuspid regurgitation (TR) and hemodynamic study with continuous doppler wave in apical 4 chamber view demonstrated TR peak velocity of $4.4 \mathrm{~m} / \mathrm{s}$ (TR peak gradient: $77 \mathrm{mmHg}$ ). Evaluation of inferior vena cava (IVC) in subcostal view was done. IVC was severely enlarged $(2 / 75 \mathrm{~cm}$ diameter) without respiratory collapse. Therefore, hemodynamic study estimated RA pressure about $20 \mathrm{mmHg}$, more compatible with chronic course of disease and indicating of more gradual increasing of RV pressure rather than acute event. Massive pericardial effusion was seen more localized in posterolateral of left ventricle $(\mathrm{LV})$ (maximum diameter: more than $3 \mathrm{~cm}$ ). There was significant respiratory variation in Doppler study of mitral valve (MV) and TV diastolic inflow velocity more than $30 \%$ (Figure 4).

Laboratory data containing hematologic, inflammatory and biomarkers were measured. D dimer was increased about $4000 \mu \mathrm{g} / \mathrm{l}$ and pro-brain natriuretic peptide (pro-BNP) $12500 \mathrm{ng} / \mathrm{d}$.

According to previous history of the COVID-19, ECG manifestation, echocardiography, chest X ray, spiral chest CT, laboratory data and abnormal hemodynamic condition, massive sub-acute PTE was highly suggested. Due to our patient hemodynamic disturbance, massive pericardial effusion and giant mass, emergent cardiac consultation with cardiovascular surgical team for surgical resection was done and the patient was taken to operating room.

Thoracotomy and median sternotomy was done and then cardiopulmonary bypass was established. Main PA was incised. Unexpectedly, out of previous suspicious, our surgeon encountered with large size tumoral solid mass with firm texture that was attached to right leaflet of PV and protruded to main PA and RVOT, which appearance was highly suggestive for sarcoma rather thrombosis. Therefore, mass completely was resected and because of tumoral involvement, right leaflet of PV removed (Figure 5). Reevaluation for residual masses in other parts was done with intraoperative echocardiography. TEE confirmed there was not any other masses in PA. Severe pulmonary insufficiency (PI) was seen after removal of right leaflet of PV. Staged operation for reconstruction of RVOT with conduit PV was postponed after preparing the result of tumor pathology and probably need for adjuvant chemotherapy. Patient had stable hemodynamic in ICU, intubated easily and discharged after 7 days without any cardiac event.

Pre-discharge TTE was done. RV and RA were severely enlarged. RV systolic function was severely impaired and severe TR and severe PI was seen. No residual mass was seen in RVOT, PV, PA and main of PA. Continuous doppler wave study showed peak TR velocity $4 \mathrm{~m} / \mathrm{s}$ and short pressure half time (PHT:24 ms) and in 2D study evidence of increased RV after load pattern was permanent. IVC plethora and engorgement without respiratory collapse was seen (Figure 6).

Pre-discharge pulmonary CT angiography was done that any mass or thrombus in pulmonary vasculature wasn't seen. In addition, abdominopelvic and brain CT for further evaluation were performed which were not abnormal.

\section{Pathology:}

Histopathological examination of resected mass showed malignant mesenchymal neoplasm composed of sheets and individual tumoral cells embedded in a desmoplastic and collagenized stroma. These cells are charactherized by enlarged, pleomorphic hyperchromatic and vesicular nuclei with frequent mitotic figures and visible nucleoli. Necrosis and hemorrhage are also present. Immunohistochemistry staining were negative for S100 and CD34 but positive for vimentin, desmin, smooth muscle actin (SMA), MNF116. In addition, about 15-20\% with Ki67 also positive in osteoclast like giant cells with CD68. (Figure 7) All this data shows 
malignant mesenchymal neoplasm compatible with undifferentiated pleomorphic sarcoma.

The patient referred to oncologist for chemotherapy. Just the day before initiation of chemotherapy, TTE was done and recurrence of tumoral mass $\left(1.5^{*} 1.5 \mathrm{~cm}\right)$ was seen in distal part of RVOT just beneath PV. Patient admitted in hospital for receiving Chemotherapy protocol with carboplatin and taxotere (docetaxel) with close monitoring of cardiac condition and hemodynamic. Unfortunately, after one course of chemotherapy patient was died in hospital with cardiogenic shock because of severe right sided heart failure.

\section{Discussion:}

Primary cardiac sarcoma are rare tumors and sarcoma of PV are very rare (4). Initial manifestation of these tumors are usually consist nonspecific symptoms of cardiopulmonary disease such as cough, dyspnea, chest pain, infrequently hemoptysis, pneumothorax and tamponade in cases with invasion to pericardium, mediastina or lung parenchyma (2-5). PA sarcoma can mimicking clinical and paraclinical findings of PTE and caused difficulties in early diagnosis and management. Some case reports representing misdiagnosis of PA sarcoma with PTE in early evaluating and for this reason the patients underwent thrombolytic therapies without any improvement and subsequent evaluations demonstrated malignant tumor of pulmonary artery $(1,6,7)$.

Chest CT scan can help differentiate PA sarcoma from PTE by low-attenuation filling defect occupying the entire luminal diameter of the main or the proximal PA, expansion of any segment of the PA, and extraluminal extension of the tumor. These image features to be suggestive of PA sarcoma (8).

Magnetic resonance imaging (MRI) is also useful diagnostic method for distinguishing PASs and PTE. On MRI, low-intensity heterogeneous features on T1-weighted images and peripheral enhancement of the thickened pulmonary artery wall on T2-weighted images and after gadolinium injection have classically been thought to support a diagnosis of PA tumor (9).

Fluorodeoxyglucose / positron emission tomography (FDG/PET) CT can distinguish PASs from PTE based on the FDG uptake with maximum standardized uptake value (SUVmax) (SUVmax of PA sarcoma is higher than of PTE) (10).

Other characteristics, such as the absence of risk factors for deep vein thrombosis and high erythrocytes sedimentation rate should raise the suspicion of a process other than PTE (1).

The only method to definitely differentiate diagnosis between PASs and PTE is histopathology (3).

Surgery is the most effective treatment of PA sarcoma $(1,7)$. Chemotherapy and radiotherapy are used and may provide longer survival, especially in patient with metastasis and incomplete resection. Endovascular stenting remains as palliative therapy for inoperable patient with obstructing tumors. Most cases of pulmonary sarcomas were diagnosed late in coarse of diseases and these made difficulties in surgical resection. Some reports shown that early diagnosis and surgical resection may provide longer survival (4-7).

Overall, PA sarcoma are very poor prognosis tumor because of late diagnosis and average of survival among reported case series were between 1.5 month to 12 months $(5,11)$. Among patients reported have longer survival, RV function were preserved and didn't mention significant increased PA pressure (5). Our patients had severe RV dysfunction in presentation and remaining of PA pressure severely high even after total resection of giant tumoral mass and in absence of any other mass or thrombosis in pulmonary arteries in pulmonary CT angiography after operation.

Patients with COVID-19 pneumonia and high D dimer value has higher prevalence PTE and myocarditis (12-15). Prior infection with COVID-19 pneumonia in our case maybe the other causes of RV failure and also masquerading sarcoma.

On the other hand, RV dysfunction due to cardiotoxicity of chemotherapy may considered as the other mechanism for progression of RV failure in our patient. Chemotherapy could induce impairment of RV structure, function and mechanistic $(16,17)$. 
Our case report emphasizes that PASs should always be included in the differential diagnosis of PTE especially if symptoms progress despite anticoagulation therapy (or any risk factor for deep vein thrombosis is not present). However, our patient underwent surgery immediately due to impaired hemodynamic and was diagnosed early compared to the time of hospitalization.

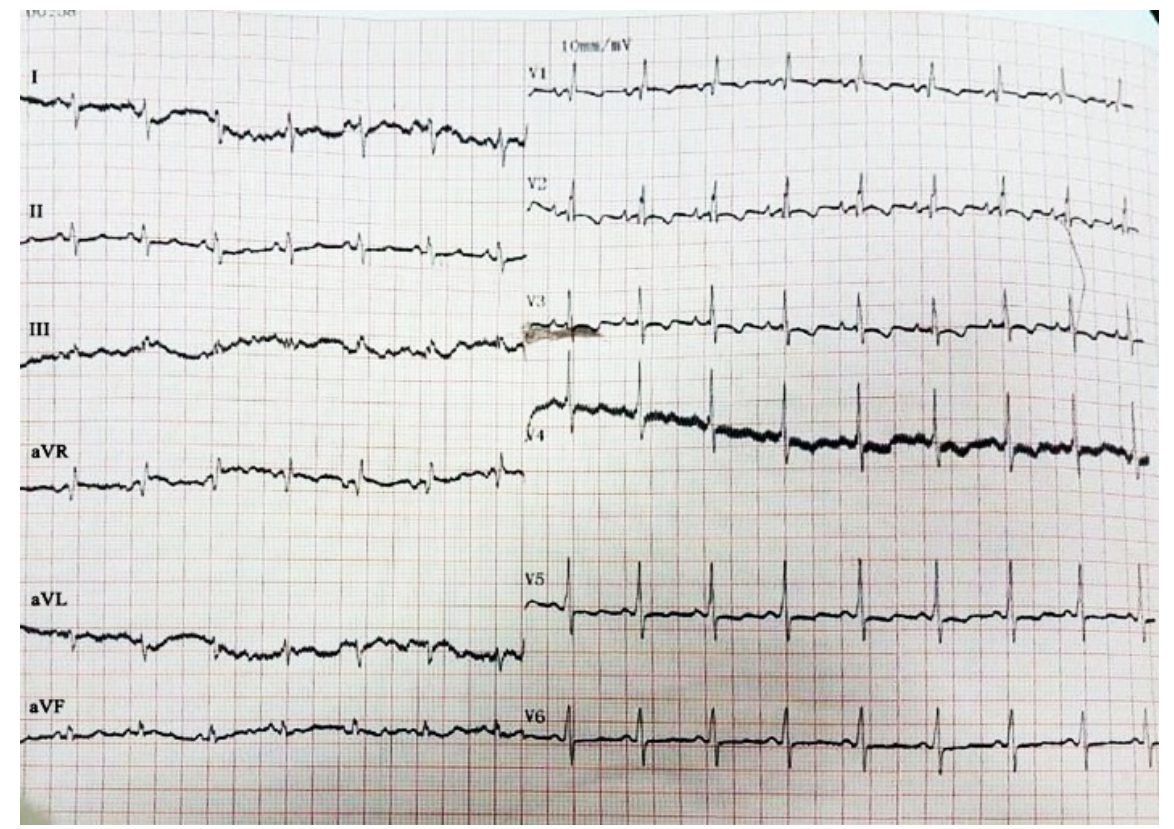

Figure 1. Electrocardiogram 


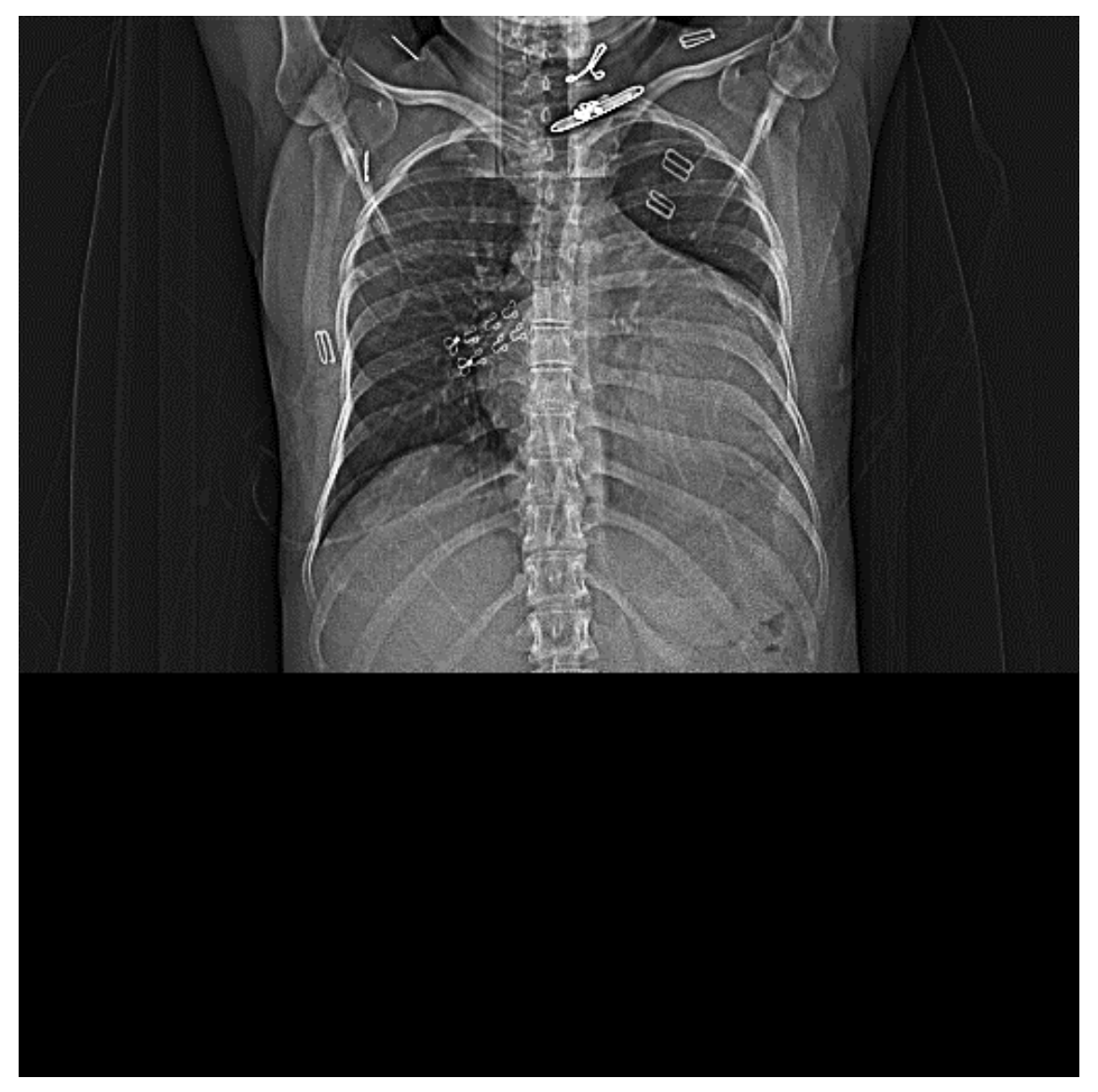

Figure 2. Chest $\mathrm{X}$ ray

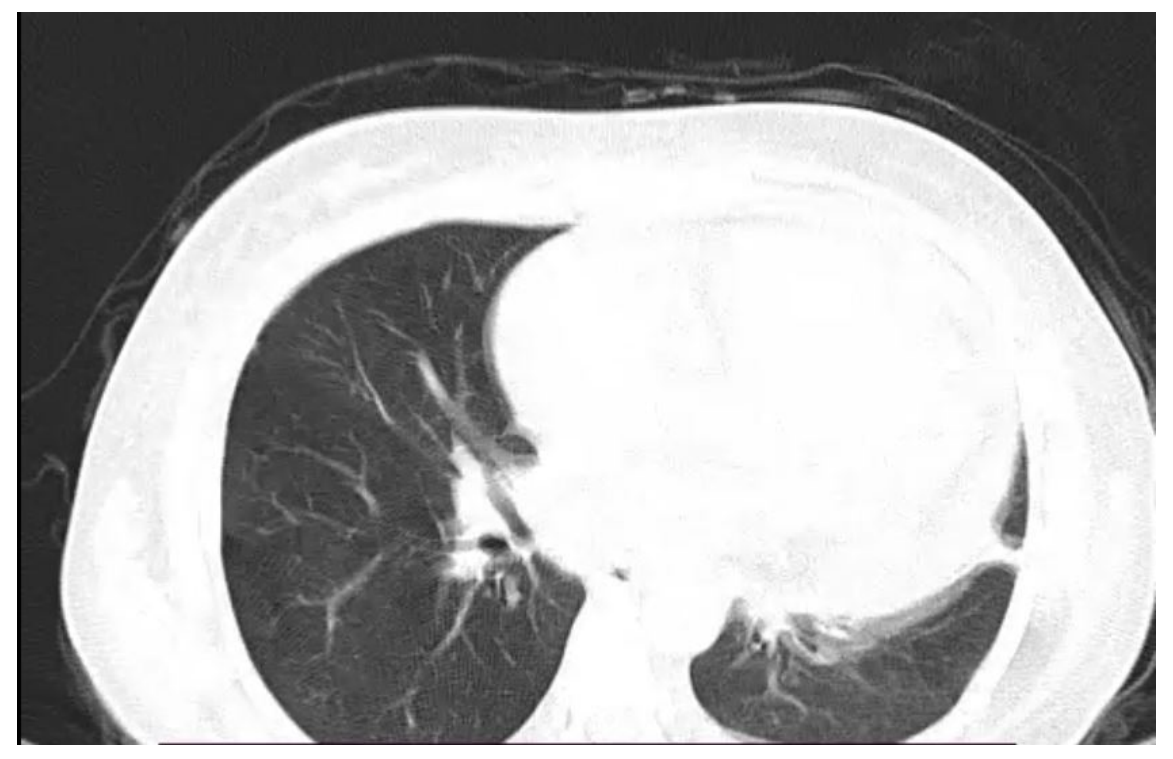




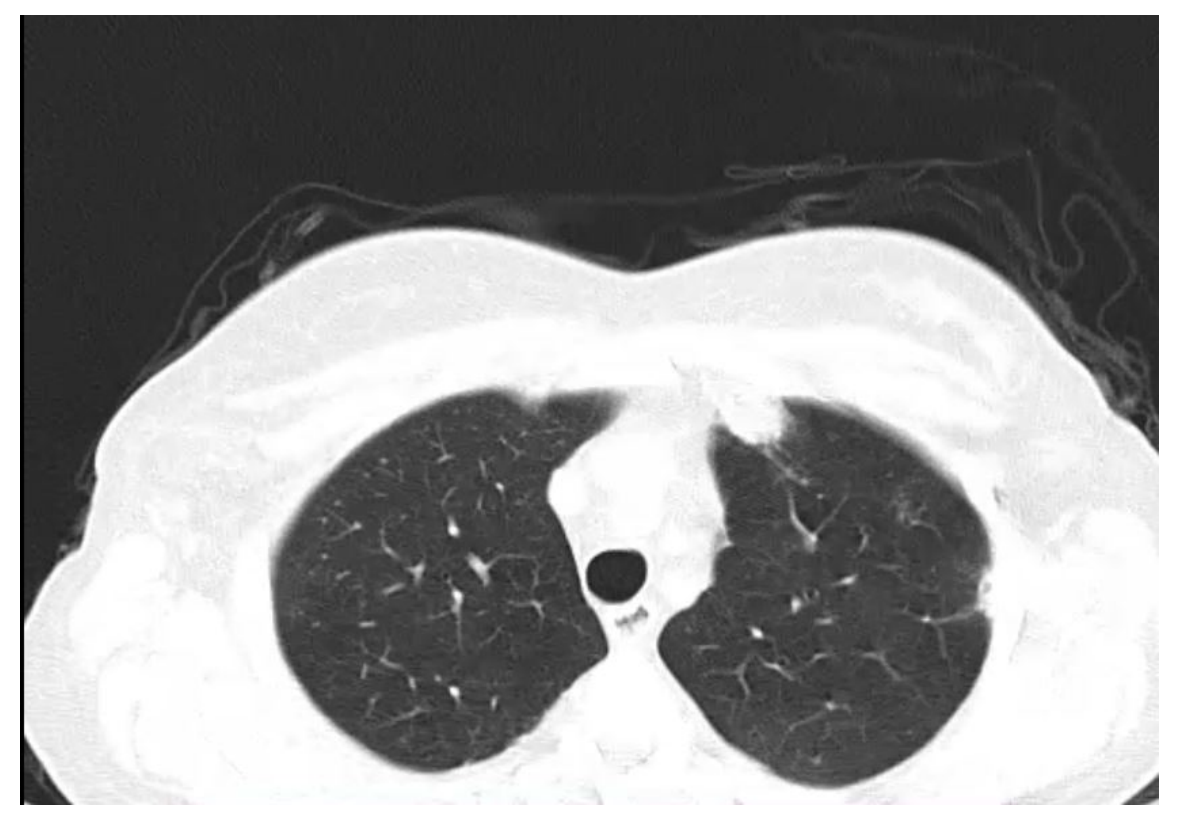

Figure 3. Spiral chest computed tomography (CT)

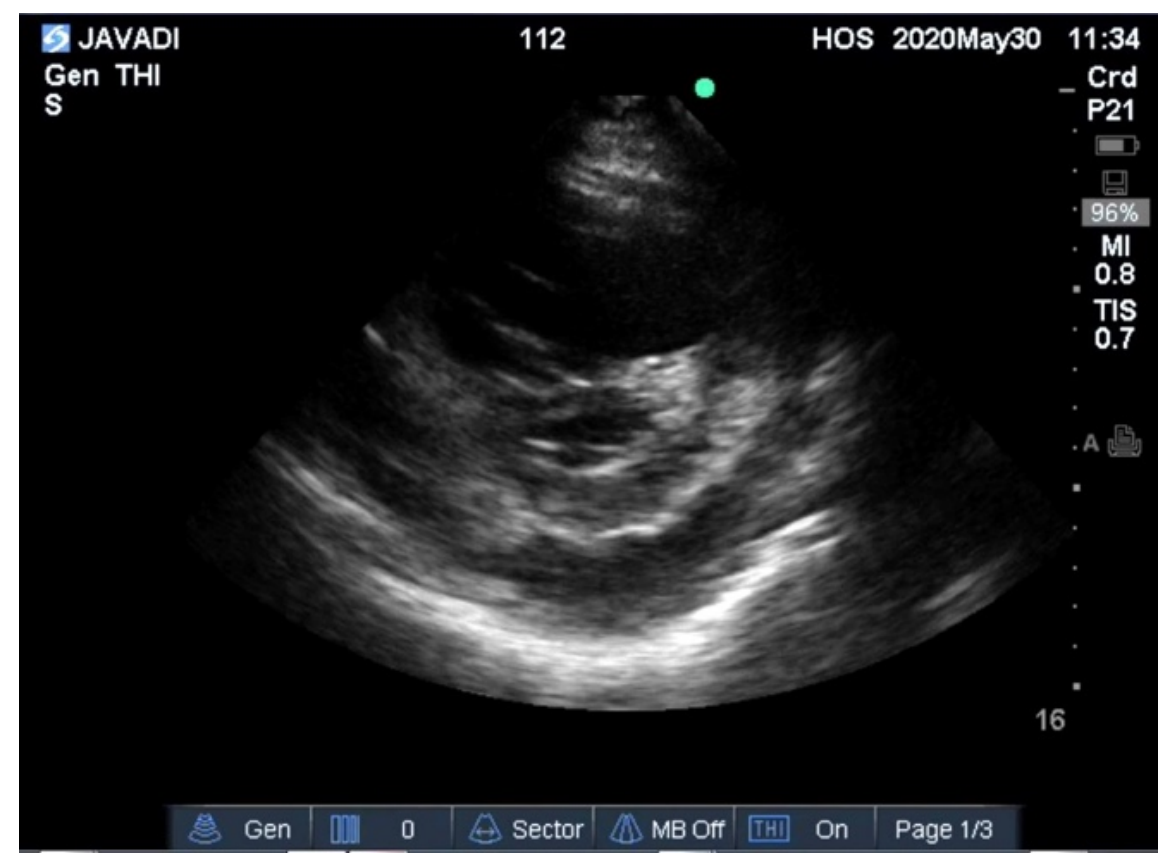



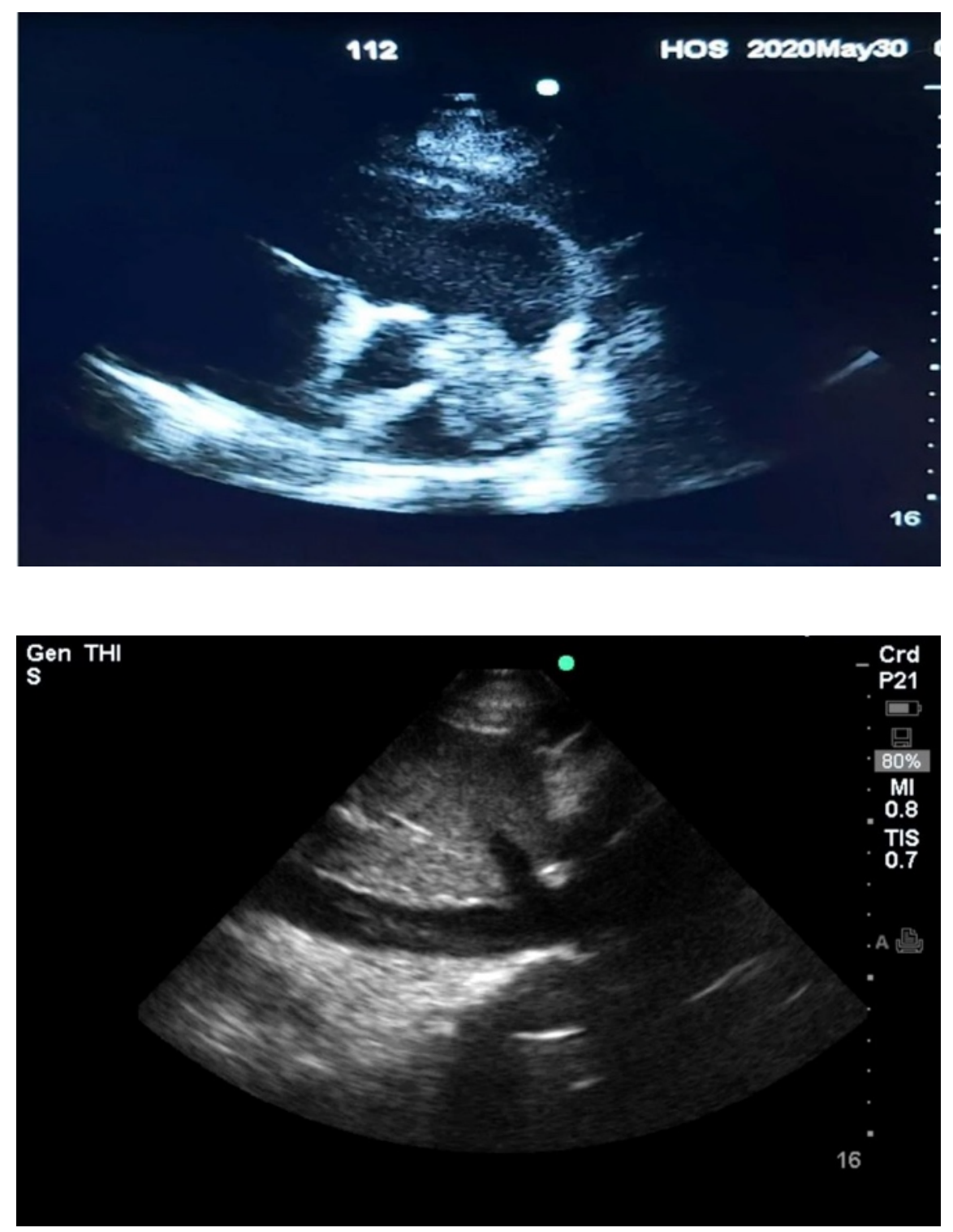

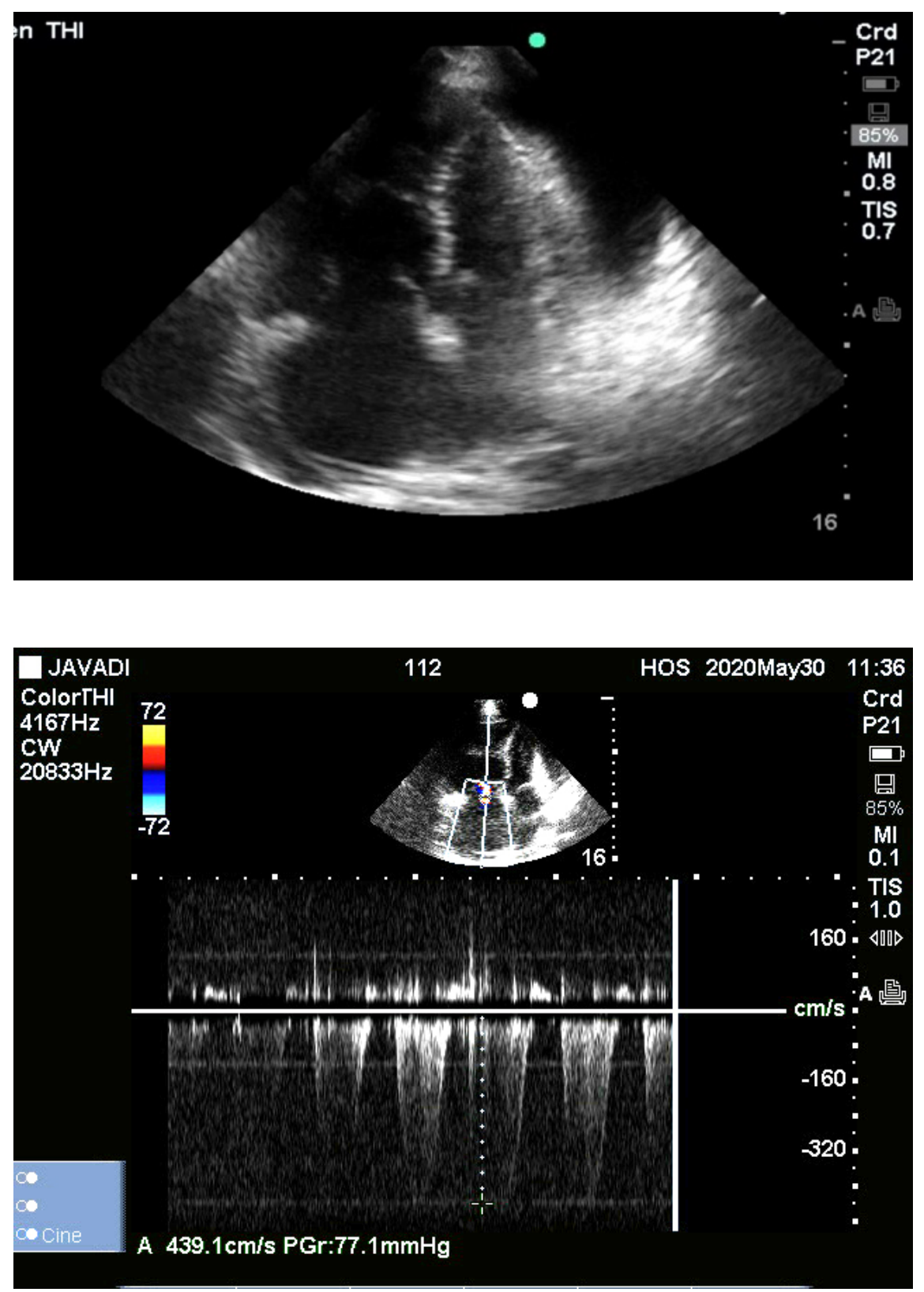

Figure 4. Transthoracic echocardiography (TTE) before surgery 


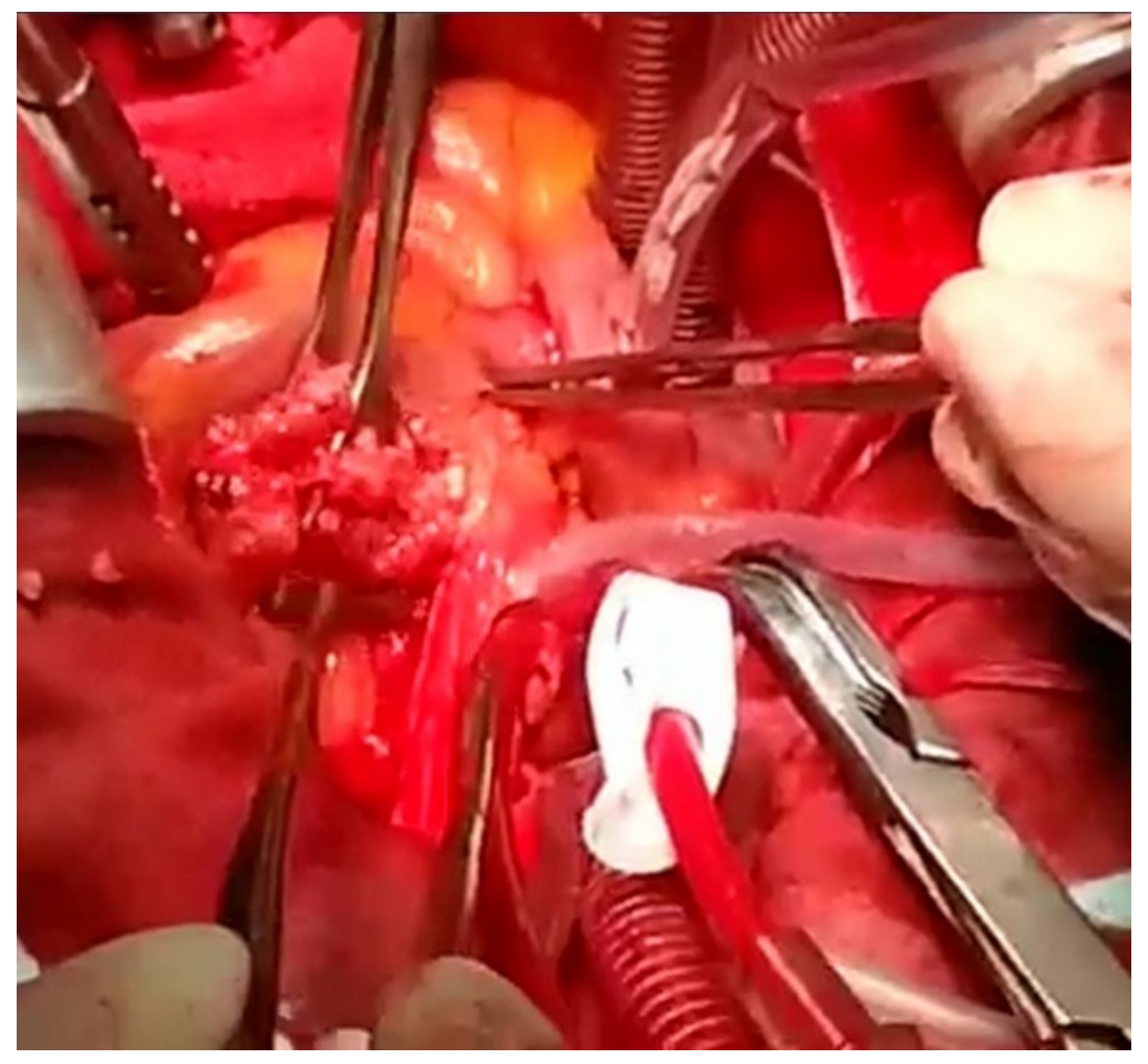




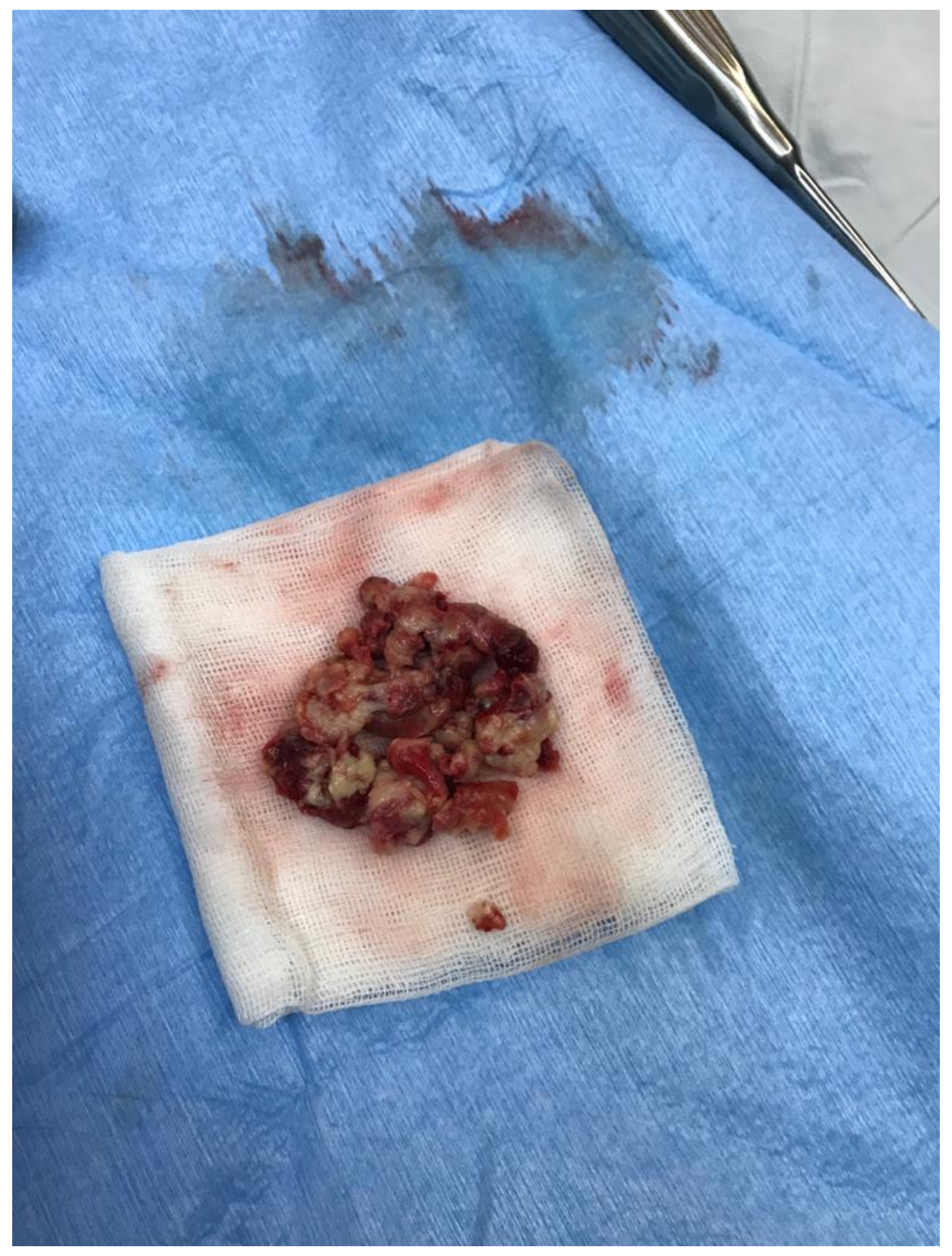

Figure 5. A: Incision of pulmonary artery with tumoral mass, B: Surgical specimen from total resection of pulmonary tumoral mass 

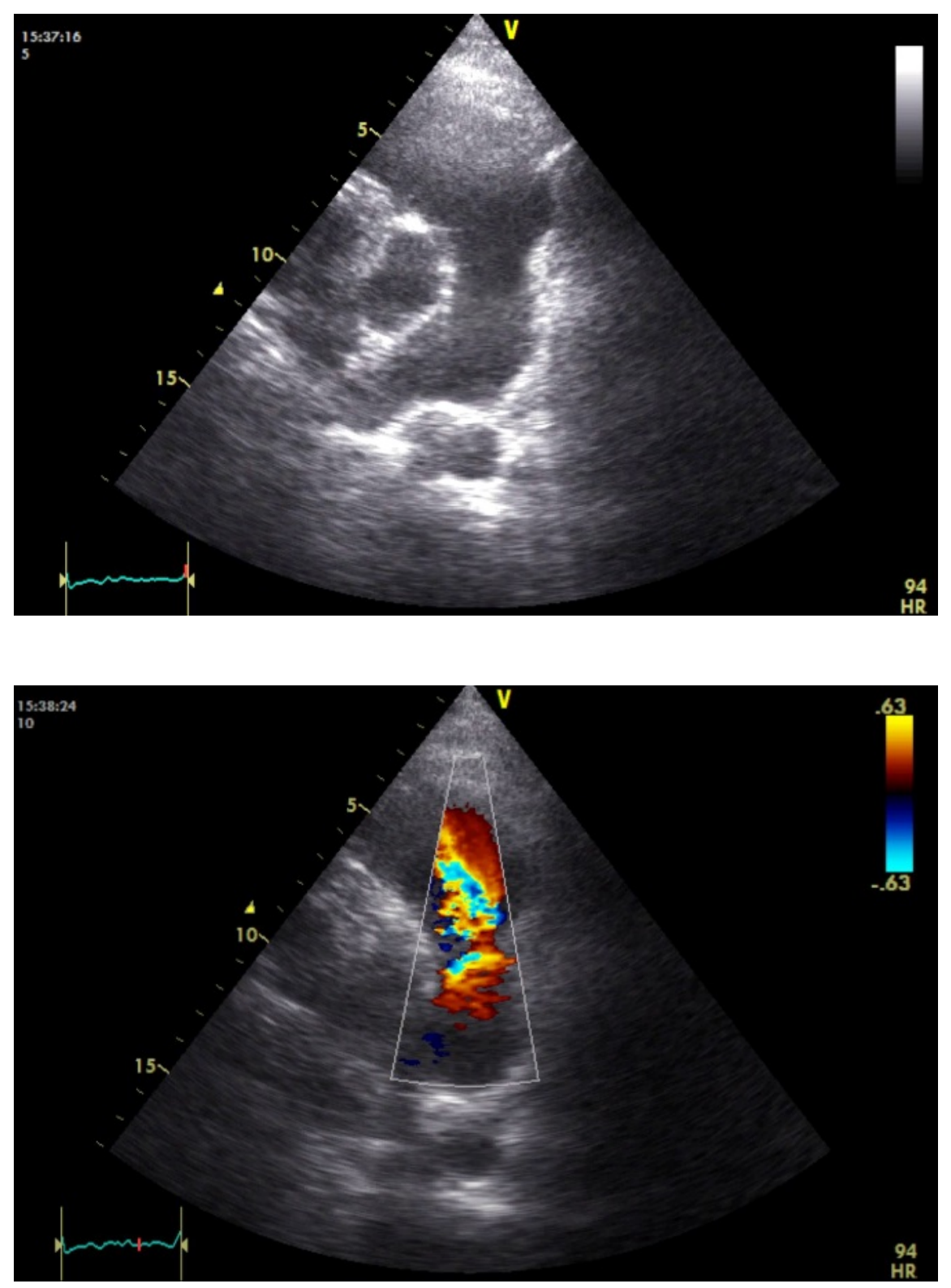


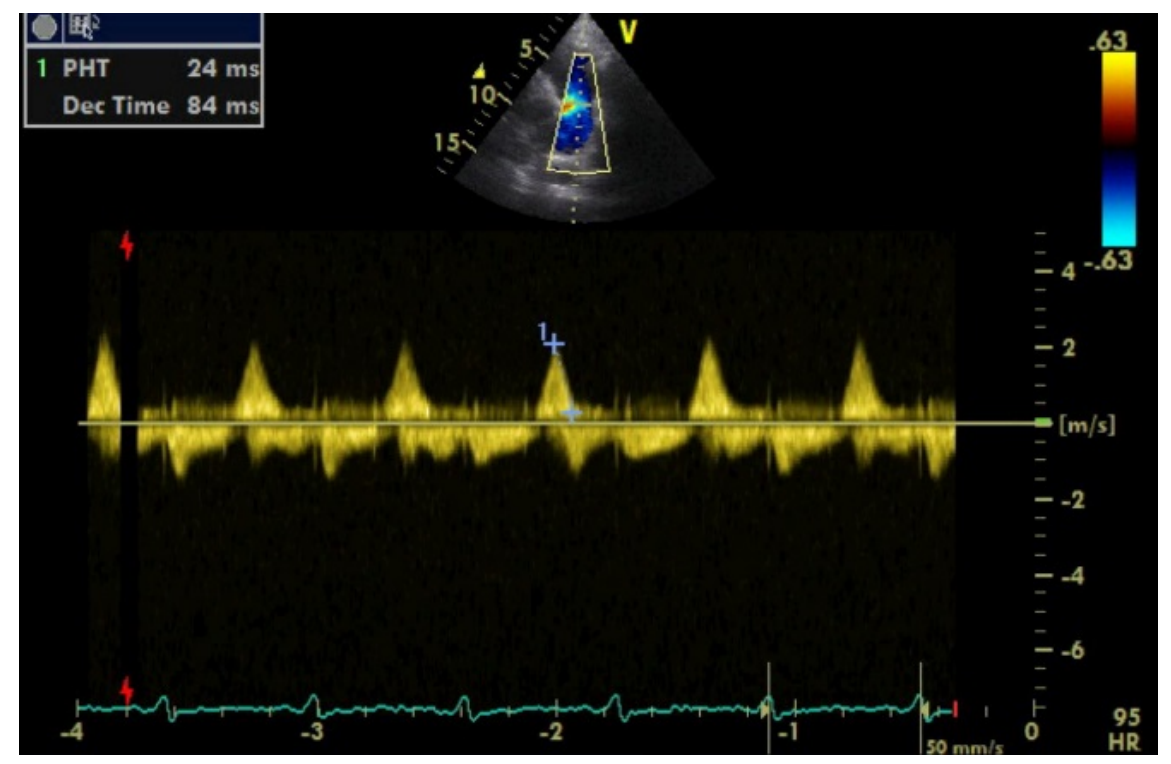

Figure 6. TTE after surgery

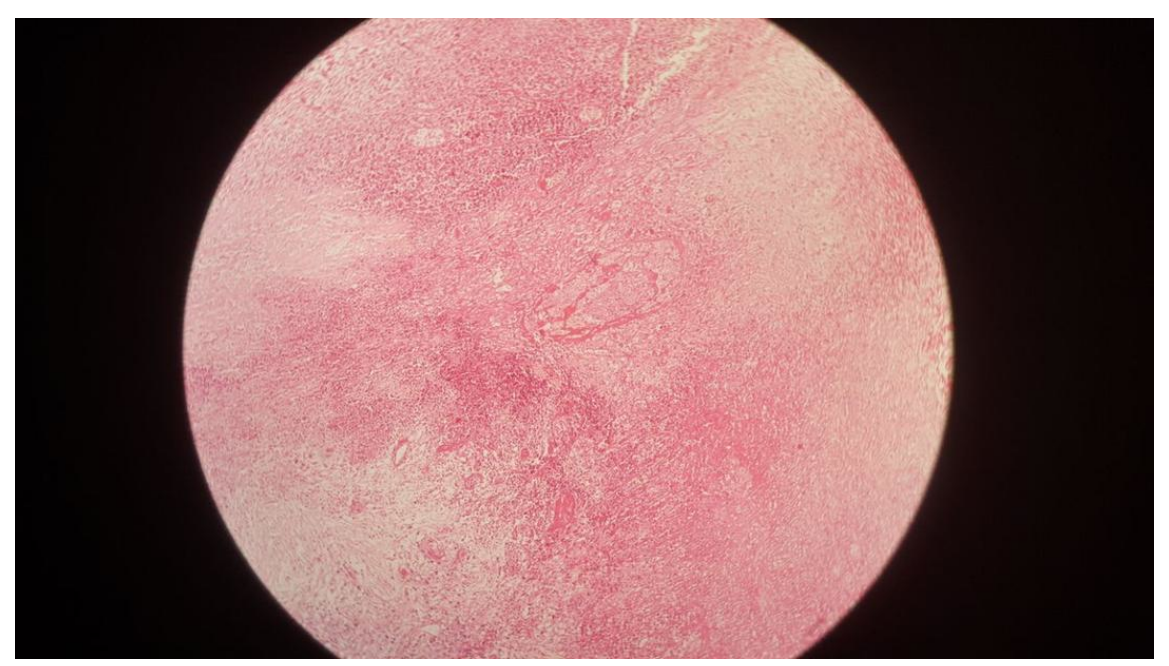



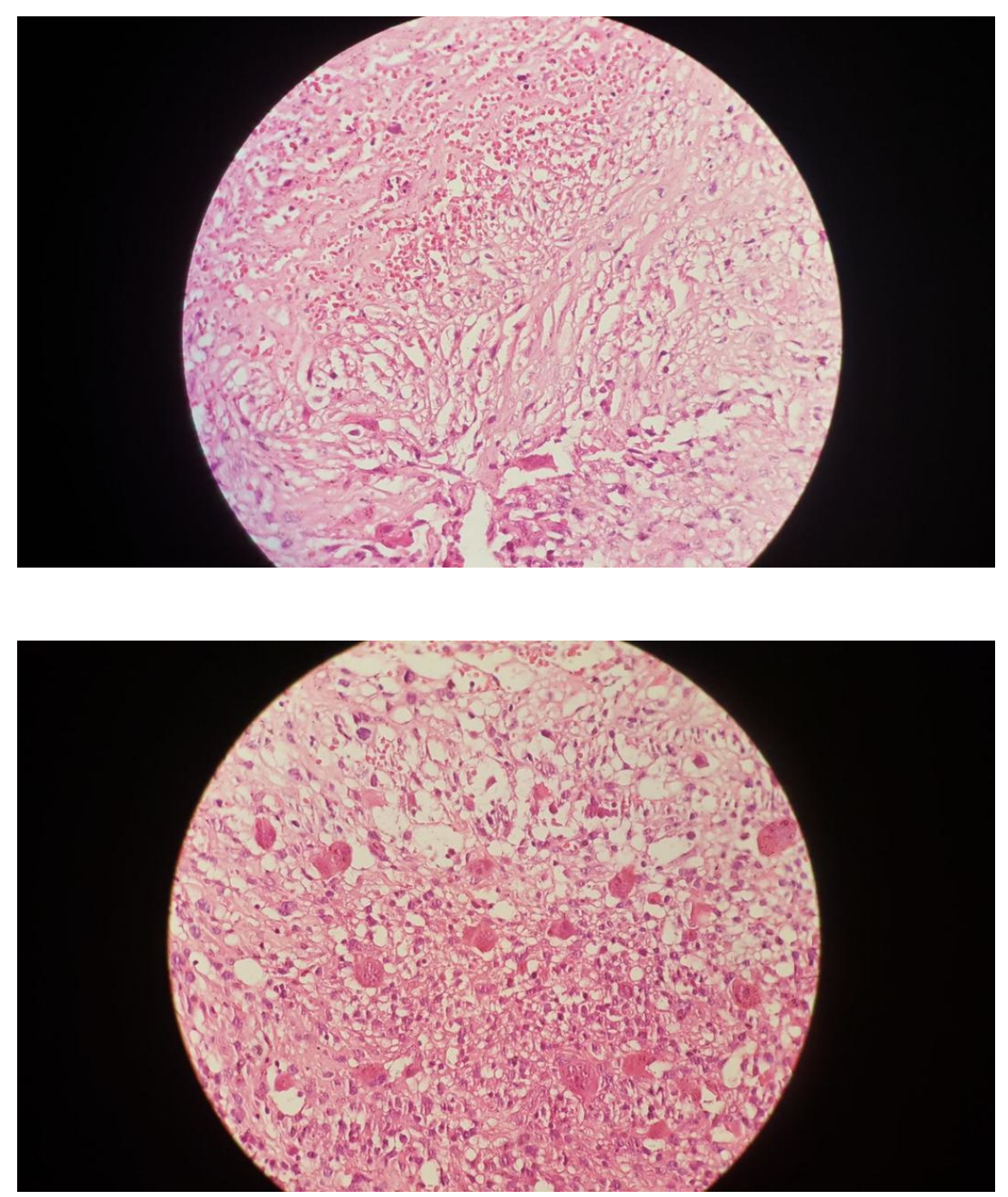

Figure 7. Histopathological examination of tissue resected

\section{Figure legends}

Figure 1. Electrocardiogram. Electrocardiogram showed sinus tachycardia, right axis deviation and right ventricle strain pattern ( $\mathrm{qR}$ in $\mathrm{V} 1$ and $\mathrm{T}$ inversion $\mathrm{V} 1-\mathrm{V} 6)$.

Figure 2. Chest $\mathbf{X}$ ray. Chest $X$ ray showed significant cardiomegaly, RV enlargement and prominent main of PA and left PA without evidence of pulmonary venous congestion.

Figure 3. Spiral chest computed tomography (CT). Chest CT showed evidence of previous COVID-19 involvement with ground glass appearance and air trapping in both lungs and also cardiomegaly and large size pericardial effusion.

Figure 4. Transthoracic echocardiography (TTE) before surgery.TTE showed severe RV enlargement with severe RV systolic dysfunction, right atrial (RA) enlargement, abnormal interventricular septal motion, severe TR, enlargement of IVC size, massive pericardial effusion and mobile mass in distal right ventricular outflow tract (RVOT) extended to main PA and has attachment to pulmonary valve (PV). 
Figure 5. A: Incision of pulmonary artery with tumoral mass, B: Surgical specimen from total resection of pulmonary tumoral mass

Figure 6. TTE after surgery. A and B: TTE parasternal short axis (PSAX) view showed no residual mass in PA after surgical resection of right leaflet of pulmonary valve and color doppler study of pulmonary valve showed severe pulmonary insufficiency (PI). C: Continuous doppler wave study of pulmonary valve after surgical resection showed severe PI and short pressure half time (PHT:24 ms).

Figure 7. Histopathological examination of tissue resected. A: Pleomorphic undifferentiated sarcoma with areas of necrosis and packed cellular areas surrounding vessels. B: In same area tumor has a vascular pattern with anastomosing irregular sinusoid channels and erythrocytes extravasation. C: In this area, there are cellular atypia, variation in cell size and shapes which consist of round cells, spindle cells, epithelioid, endothelial like and multi nuclear giant cells.

\section{References}

1. Toporcer T, Martincek M, Mistrikova L, Sabol F. Primary pulmonary valve sarcoma involving pulmonary artery and right ventricular outflow tract. Cor et Vasa. October 2015; 57(5):e371-e376.

2. S. Kanjanauthai, T. Kanluen, C. Ray, Pulmonary artery sarcoma masquerading as saddle pulmonary embolism. Heart, Lung \& Circulation. 17 (2008) 417-419.

3. E. Levy, A. Korach, G. Amir, et al., Undifferentiated sarcoma of the pulmonary artery mimicking pulmonary thromboembolic disease. Heart, Lung \& Circulation.15 (2006) 62-63.

4. Huo L, Moran CA, Fuller GN, Gladish G, Suster S. Pulmonary artery sarcoma: a clinicopathologic and immunohistochemical study of 12 cases. Am J Clin Pathol. 2006 Mar;125(3):419-24.

5. Morreau SP, Haydock DA. Prolonged Survival of Pulmonary Artery Sarcoma After Aggressive Surgical Resection. Ann Thorac Surg. 2017 Jan;103(1):e21-e23.

6. Mahajan A, Rekhi B, Laskar S, Bajpai J, Jayasree L, Thakur MH. Primary pulmonary artery sarcoma masquerading as pulmonary thromboembolism: a rare diagnosis unveiled. Clin Sarcoma Res. 2017 Jul $1 ; 7: 13$

7. Kim JB, Kim SH, Lim SY, Roh SY, Cho GY, Song HJ, Park S, Lee J, Kim SJ. Primary angiosarcoma of the pulmonary trunk mimicking pulmonary thromboembolism. Echocardiography. 2010 Feb;27(2):E236.

8. C.A. Yi, K.S. Lee, Y.H. Choe, et al., Computed tomography in pulmonary artery sarcoma: distinguishing features from pulmonary embolic disease, Journal of Computer Assisted Tomography 28 (2004) $34-39$.

9. N. Girard, C. Triby-Moreau, S. Benabidallah, et al., Pulmonary artery sarcoma, a paradigm of orphan thoracic oncology, Presse Med 38 (2009) 1167-1170.

10. K. Ito, K. Kubota, M. Morooka, et al., Diagnostic usefulness of 18F-FDG PET/CT in the differentiation of pulmonary artery sarcoma and pulmonary embolism, Annals of Nuclear Medicine 23 (2009) 671-676.

11. Said SM, Sundt TM 3rd, Garces YI, Wigle DA. 5-year survival after multiple repeat metastasectomy for pulmonary artery angiosarcoma. Ann Thorac Surg. 2011Apr;91(4):e4951.

12. Alonso-Fernández A, Toledo-Pons N, Cosío BG, Millán A, Calvo N, Ramón L, de Mendoza SH, Morell-García D, Bauça-Rossello JM, Núñez B, Pons J. Prevalence of pulmonary embolism in patients with COVID-19 pneumonia and high D-dimer values: A prospective study. PloS one. 2020 Aug 25;15(8):e0238216.

13. Cristina Basso, Ornella Leone, Stefania Rizzo, Monica De Gaspari, Allard C van der Wal, MarieChristine Aubry, Melanie C Bois, Peter T Lin, Joseph J Maleszewski, James R Stone, Pathological features of COVID-19-associated myocardial injury: a multicentre cardiovascular pathology study, European Heart Journal, Volume 41, Issue 39, 14 October 2020, Pages 3827-3835.

14. Helms J, Tacquard C, Severac F, et al. High risk of thrombosis in patients with severe SARS-CoV-2 infection: a multicenter prospective cohort study. Intensive Care Med. 2020;46(6):1089-1098.

15. Park JF, Banerjee S, Umar S. In the eye of the storm: the right ventricle in COVID-19. Pulmonary Circulation. July 2020. doi:10.1177/2045894020936660 
16. Jahangir, E., Harinstein, M.E., Murthy, V.L. et al. The forgotten right ventricle in cardio-oncology. J. Nucl. Cardiol. 27, 2164-2166 (2020).

17. Tadic M, Cuspidi C, Hering D, Venneri L, Danylenko O. The influence of chemotherapy on the right ventricle: did we forget something? Clin Cardiol. 2017 Jul;40(7):437-443. Epub 2017 Feb 13.
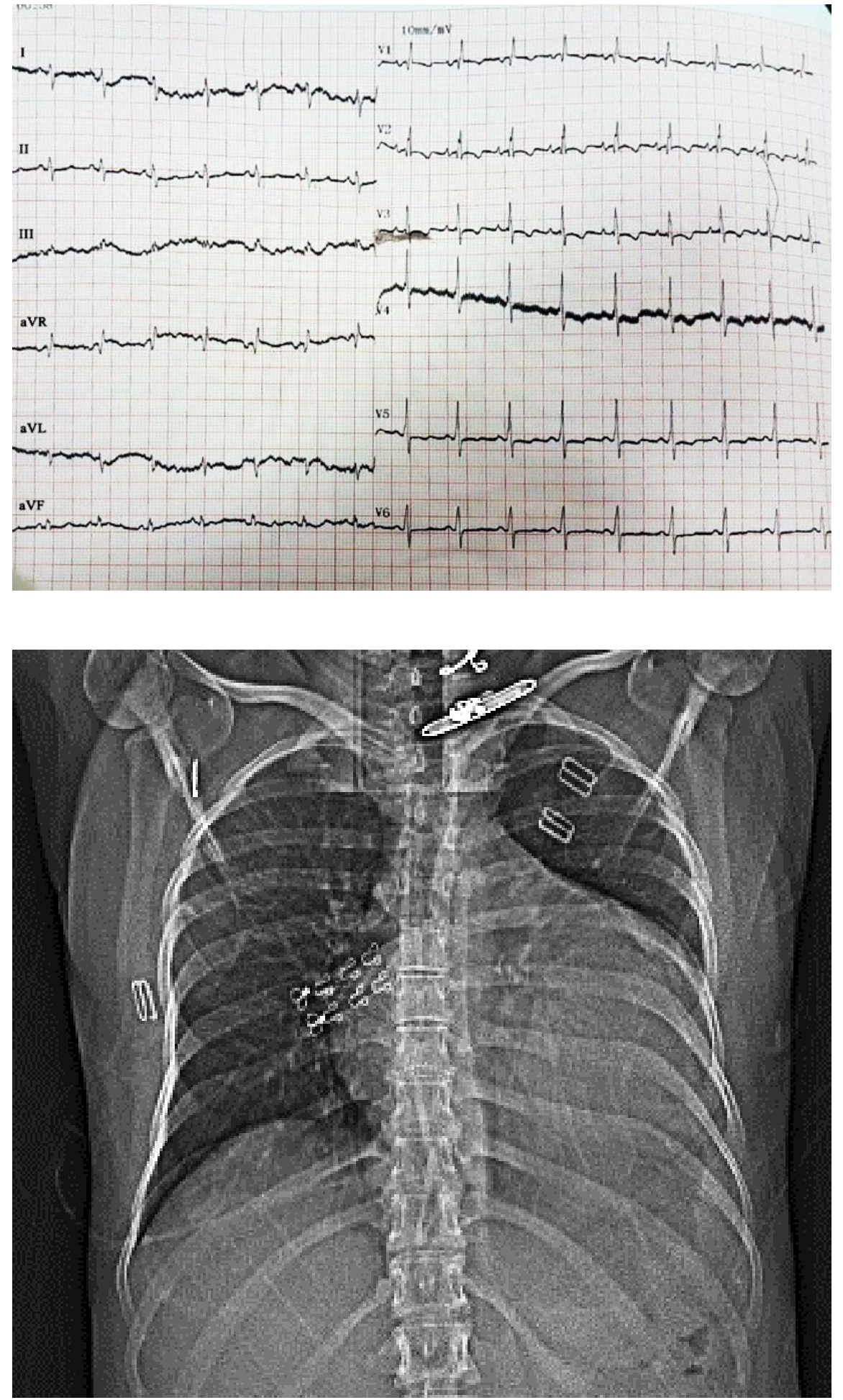

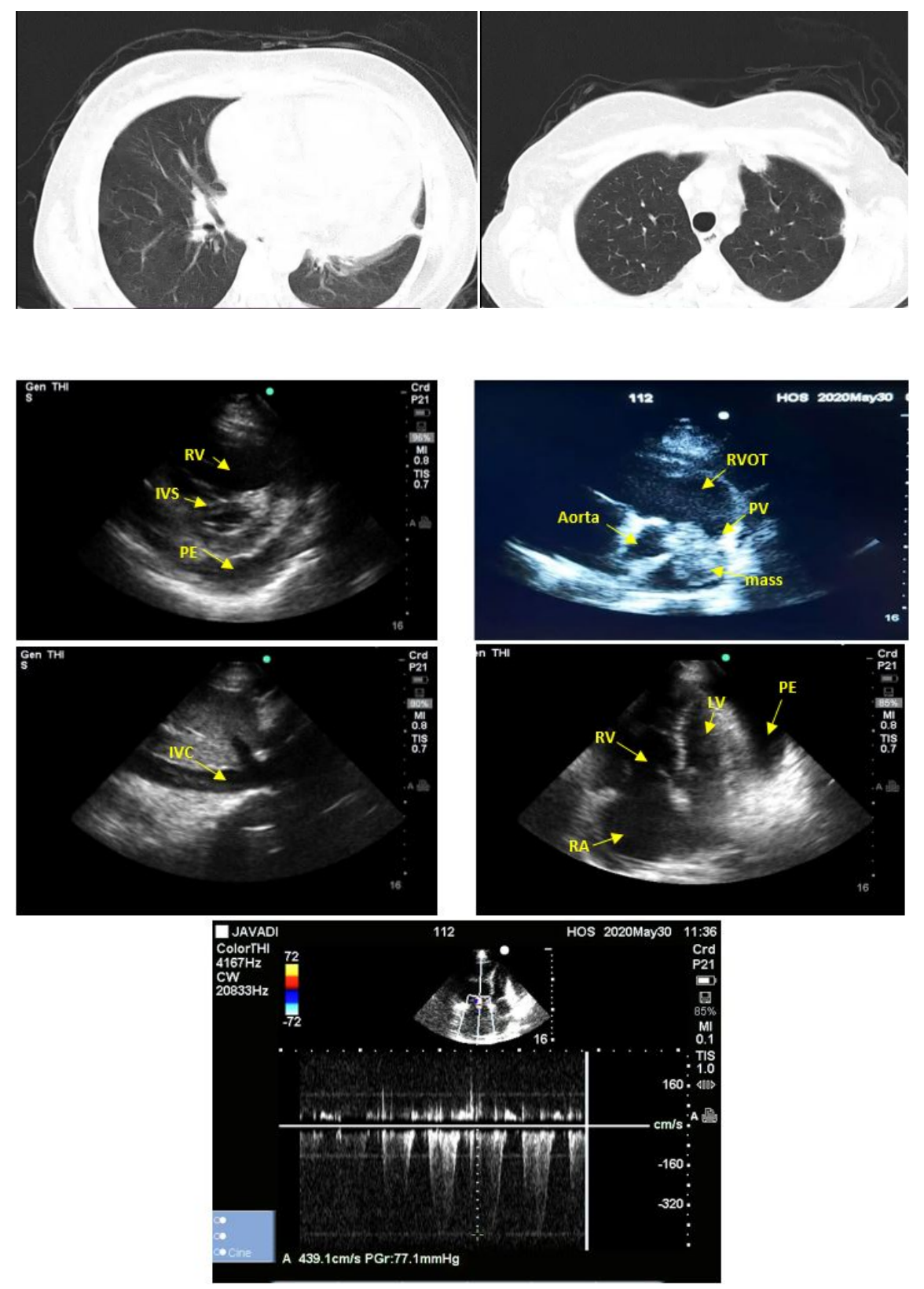

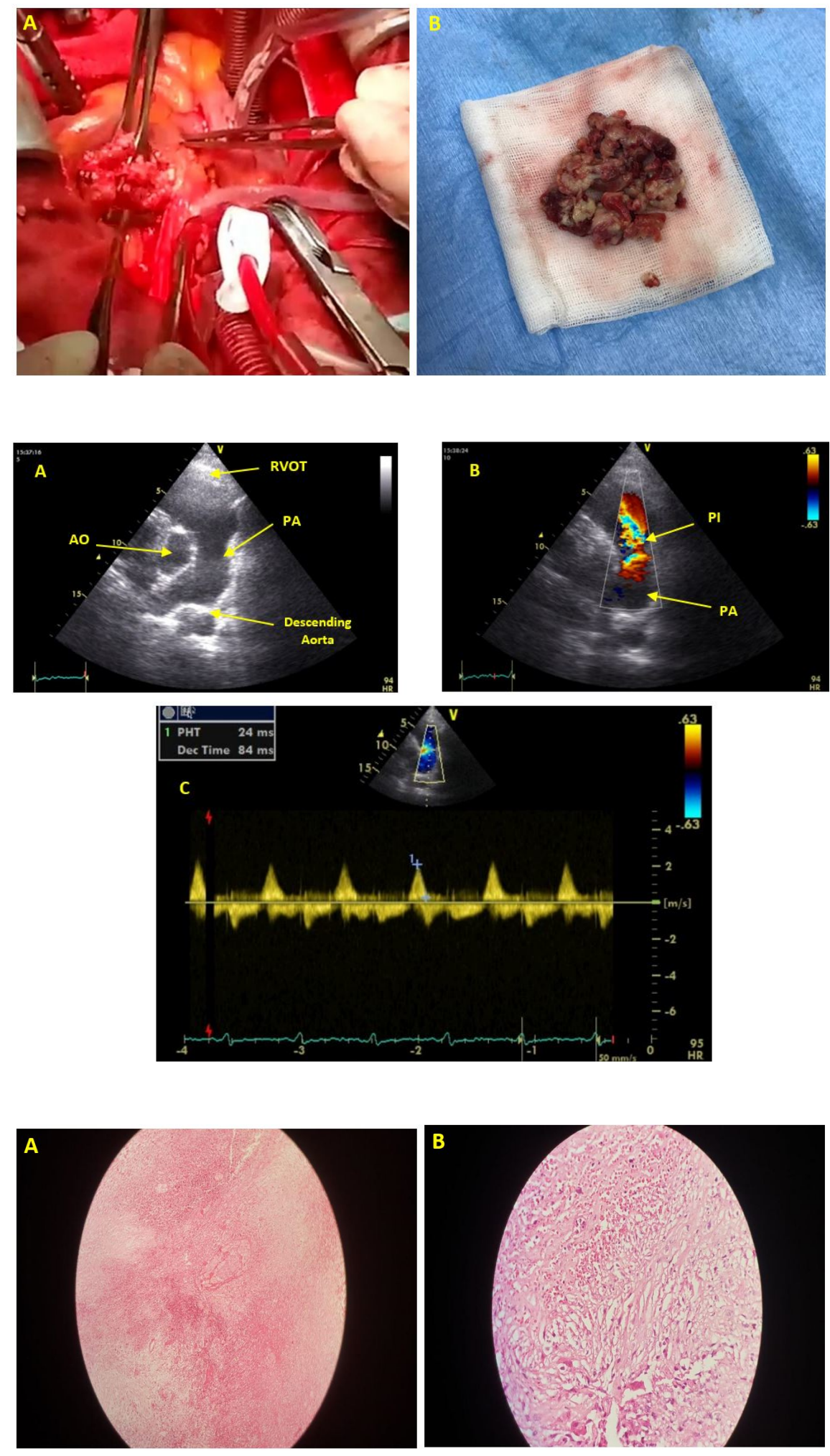